\title{
Mirabegron Improves Nocturia, Nocturia-Associated Quality of Life, and Sleep Quality in Female Patients with Overactive Bladder
}

\author{
Yoshida $\mathbf{M}^{2}$, Gotoh $\mathbf{M}^{2}$, Kageyama $\mathrm{S}^{3}$, Kato $\mathrm{K}^{4}$, \\ Matsukawa $\mathbf{Y}^{2}$, Narushima $\mathbf{M}^{5}$ and Study Group of \\ N-QOL \\ ${ }^{1}$ Department of Urology, National Center for Geriatrics \\ and Gerontology, J apan \\ ${ }^{2}$ Department of Urology, Nagoya University Graduate \\ School of Medicine, J apan \\ ${ }^{3}$ Kageyama Urology Clinic, J apan \\ ${ }^{4}$ Department of Female Urology, Red Cross Nagoya First \\ Hospital, J apan \\ ${ }^{5}$ Department of Urology, Meitetsu Hospital, J apan \\ *Correspondling author: Yoshida M, Department of \\ Urology, National Center for Geriatrics and Gerontology, \\ 7-430 Morioka-cho, Obu, Aichi 474-8511, J apan
}

Received: December 22, 2016; Accepted: February 27, 2017; Published: March 03, 2017

\begin{abstract}
Objective: To evaluate the effects of mirabegron, a $\beta 3$-adrenoceptor agonist, on nocturia, nocturia-associated Quality of Life (QOL), and sleep quality in female patients with Overactive Bladder (OAB) patients.

Materials and Methods: This prospective, multicenter study involved 60 female patients with $\mathrm{OAB}$ who experienced two or more nocturnal voids per night and visited 1 of 16 medical institutes. Mirabegron ( $50 \mathrm{mg} /$ day) was administered for 12 weeks. The frequency-volume chart and various questionnaires (International Prostate Symptom Score, QOL index, OAB Symptom Score, Pittsburgh Sleep Quality Index [PSQI], and Nocturia QOL [N-QOL]) were examined before therapy and at 4, 8, and 12 weeks after mirabegron administration. The Wilcoxon signed rank test or paired t-test was used to compare the parameters before and after treatment.
\end{abstract}

Results: The mean patient age was $75.3 \pm 6.8$ years. In total, 58 patients were analyzed; 2 withdrew consent and were excluded. After 12 weeks of mirabegron treatment, there was a significant improvement in the nocturnal voiding frequency, nocturnal urine volume per void, urine volume of the first nocturnal void, and N-QOL scores. The mean hours of undisturbed sleep increased after 12 weeks of treatment, although this increase was not statistically significant. The PSQI was significantly improved after 12 weeks of treatment. Side effects were seen in five patients (8.6\%); however, all side effects were mild.

Conclusion: Mirabegron improved the nocturnal voiding frequency by increasing the nocturnal bladder capacity, thereby improving QOL by increasing sleep quality in female patients with nocturia.

Keywords: Mirabegron; Nocturia; Overactive bladder; Quality of life; Sleep disorder

\section{Ablbreviations}

OAB: Overactive Bladder; QOL: Quality of Life; N-QOL: Nocturia Quality of Life Questionnaire; OABSS: Overactive Bladder Symptom Score; I-PSS: International Prostatic Symptom Score; PSQI, Pittsburgh Sleep Quality Index; HUS: Hours of Undisturbed Sleep

\section{Introduction}

In 2002, the International Continence Society defined nocturia as "waking one or more times to void during the night" [1]. Nocturia has been associated with urinary bladders to rage disorders, as observed in patients with benign prostatic hyperplasia and Overactive Bladder $(\mathrm{OAB})$; it has also been associated with polyuria, nocturnal polyuria, and sleep disorders [2]. Nocturia significantly compromises patients' Quality of Life (QOL) [3,4] and is usually the most problematic symptom among a variety of lower urinary tract symptoms [5]. Although treatment for nocturia depends on the underlying pathology, nocturia is reportedly improved by the administration of anticholinergic agents, which are one of the first-line drugs for treatment of $\mathrm{OAB}[6-8]$.
Mirabegron, a selective $\beta 3$-adrenoceptor agonist, was recently developed as a therapeutic agent for OAB. Relaxation of the human bladder during the period of urine storage occurs due to binding of noradrenaline released from the sympathetic nerves to $\beta 3$ adrenoceptors expressed in the smooth muscle of the bladder [9$12]$. Mirabegron binds to $\beta 3$-adrenoceptors, resulting in enhanced relaxation of the bladder during urine storage and an increased bladder capacity, thus improving frequency, urgency, and urgency incontinence associated with $\mathrm{OAB}$ [13]. Usefulness of mirabegron in relief of $\mathrm{OAB}$ symptoms has already been investigated in many studies [14-18], but few reports have evaluated the effectiveness of mirabegron in patients with nocturia.

In this study, we investigated the efficacy and safety of mirabegron for nocturia in female patients with OAB. The Nocturia QOL Questionnaire (N-QOL) [19,20], a QOL questionnaire specific for nocturia, was used to assess the impact of mirabegron on QOL.

\section{Materials and Methods}

This study was initiated after approval from the institutional
Austin J Urol - Volume 4 Issue 1 - 2017

ISSN: 2472-3606 | www.austinpublishing group.com

Yoshida et al. (c) All rights are reserved
Citation: Yoshida M, Gotoh M, Kageyama S, Kato K, Matsukawa Y, Narushima M. Mirabegron Improves Nocturia, Nocturia-Associated Quality of Life, and Sleep Quality in Female Patients with Overactive Bladder. Austin J Urol. 2017; 4(1): 1054 
Table 1: Effects of mirabegron on parameters of frequency-volume chart.

\begin{tabular}{|c|c|c|c|}
\hline & Before treatment & After 12 weeks of mirabegron ${ }^{\dagger}$ & $P$ value \\
\hline \multicolumn{4}{|l|}{ Frequency-volume chart parameters } \\
\hline Nocturnal voiding frequency & $2.5 \pm 1.1$ & $1.9 \pm 1.0$ & $0.0128^{\star \dagger}$ \\
\hline Daytime voiding frequency & $8.9 \pm 2.2$ & $7.4 \pm 1.8$ & $<0.0001^{* *+1+}$ \\
\hline 24-h voiding frequency & $11.3 \pm 2.5$ & $9.3 \pm 1.9$ & $<0.0001^{*+*+1}$ \\
\hline Daytime urine volume $(\mathrm{ml})$ & $894.0 \pm 283.6$ & $885.1 \pm 322.4$ & $0.1763^{\S}$ \\
\hline Nocturnal urine volume (ml) & $612.3 \pm 259.5$ & $556.7 \pm 230.4$ & $0.1035^{\S}$ \\
\hline 24-h urine volume (ml) & $1506.3 \pm 451.5$ & $1441.7 \pm 459.5$ & $0.0689^{\S}$ \\
\hline Nocturnal polyuria index (\%) & $40.3 \pm 10.6$ & $38.8 \pm 10.6$ & $0.6379^{\S}$ \\
\hline Urine volume per void (ml) & $141.1 \pm 49.1$ & $161.4 \pm 69.6$ & $0.0032^{* \star 8}$ \\
\hline Nocturnal urine volume per void (ml) & $263.0 \pm 98.9$ & $318.9 \pm 163.6$ & $0.0374^{\star \S}$ \\
\hline Daytime urine volume per void (ml) & $107.4 \pm 35.0$ & $124.7 \pm 51.9$ & $0.0054^{* \star \S}$ \\
\hline Urine volume of first nocturnal void (ml) & $184.5 \pm 83.6$ & $228.1 \pm 110.2$ & $0.0495^{\star \S}$ \\
\hline Hours of undisturbed sleep & $160.6 \pm 74.3$ & $203.8 \pm 96.4$ & $0.0632^{\S}$ \\
\hline \multicolumn{4}{|c|}{ †50 mg of oral mirabegron once daily; ${ }^{\ddagger}$ Wilcoxon signed rank test; ${ }^{5}$ paired $t$-test } \\
\hline \multicolumn{4}{|l|}{${ }^{\star} P<0.05,{ }^{* \star} P<0.01,{ }^{\star * \star *} P<0.001$} \\
\hline
\end{tabular}

Table 2: Effects of mirabegron on International Prostatic Symptom Score (I-PSS) and Overactive Bladder Symptom Score (OABSS).

\begin{tabular}{|c|c|c|c|}
\hline & Before treatment & After 12 weeks of mirabegron ${ }^{\dagger}$ & $P$ value \\
\hline \multicolumn{4}{|l|}{ I-PSS } \\
\hline Total score & $12.4 \pm 5.8$ & $7.3 \pm 4.8$ & $<0.0001^{\star \star \star}$ \\
\hline Voiding score & $3.7 \pm 3.5$ & $2.7 \pm 2.9$ & $0.0132^{*}$ \\
\hline Storage score & $7.2 \pm 3.0$ & $3.9 \pm 1.6$ & $<0.0001^{\star \star \star}$ \\
\hline Post-micturition score & $1.5 \pm 1.6$ & $0.9 \pm 1.2$ & $0.00132^{* *}$ \\
\hline Nighttime frequency & $2.5 \pm 0.9$ & $1.9 \pm 0.8$ & $<0.0001^{\star \star *}$ \\
\hline I-PSS quality of life & $4.8 \pm 1.1$ & $3.0 \pm 1.5$ & $<0.0001^{\star \star *}$ \\
\hline \multicolumn{4}{|l|}{ OABSS } \\
\hline Total score & $9.0 \pm 2.4$ & $4.5 \pm 2.2$ & $<0.0001^{* \star *}$ \\
\hline Daytime frequency & $1.0 \pm 0.5$ & $0.8 \pm 0.4$ & $<0.0018^{\star \star \star}$ \\
\hline Nighttime frequency & $2.5 \pm 0.8$ & $1.8 \pm 0.8$ & $<0.0001^{* * *}$ \\
\hline Urgency & $3.3 \pm 1.0$ & $1.2 \pm 1.2$ & $<0.0001^{\star \star *}$ \\
\hline Urgency incontinence & $2.1 \pm 1.6$ & $0.7 \pm 0.9$ & $<0.0001^{\star \star *}$ \\
\hline \multicolumn{4}{|c|}{ Differences compared using the Wilcoxon signed rank test } \\
\hline \multicolumn{4}{|c|}{ †50 mg of oral mirabegron once daily } \\
\hline${ }^{\star} P<0.05,{ }^{* *} P<0.01$, & & & \\
\hline
\end{tabular}

review boards of each of the 16 individual medical centers that participated in this study. All participants provided written informed consent, and the study protocol conformed to the provisions of the Declaration of Helsinki (as revised in Edinburgh 2000).

The patients comprised 60 female outpatients with $\mathrm{OAB}$ aged $\geq 50$ years with an average of two or more nocturnal voids per night according to a voiding diary. All patients were able to complete the questionnaire by themselves, go to the bathroom without assistance, and measure the voided volume by themselves. The exclusion criteria were serious cardiac diseases and arrhythmias; serious hepatic diseases, renal impairment, and glaucoma; having taken anticholinergic agents for treatment of $\mathrm{OAB}$ within the previous 3 months; hypokalemia; dysuria; $\geq 100 \mathrm{~mL}$ of residual urine; polyuria with a mean daily voiding volume of $\geq 40 \mathrm{~mL} / \mathrm{kg}$; an indwelling catheter or intermittent self-urination; and urinary tract infection, urinary calculi, interstitial cystitis, and/or bladder tumors.

All enrolled patients were administered $50 \mathrm{mg}$ of mirabegron after a meal once daily for 12 weeks.

The patients completed questionnaires on their symptoms (OAB Symptom Score [OABSS], International Prostatic Symptom Score [I-PSS], I-PSS-QOL score, N-QOL, and Pittsburgh Sleep Quality Index [PSQI]) before treatment and at 4, 8, and 12 weeks after the start of treatment. In addition, the patients kept a voiding diary for 3 days before each study visit. At each visit, the residual urine volume, blood pressure, and pulse rate were measured, and medication 
Table 3: Effects of mirabegron on Nocturia Quality of Life Questionnaire (N-QOL) and Pittsburgh Sleep Quality Index (PSQI).

\begin{tabular}{|c|c|c|c|}
\hline & Before treatment & After 12 weeks of mirabegron ${ }^{\dagger}$ & $P$ value \\
\hline \multicolumn{4}{|l|}{ N-QOL } \\
\hline Total score & $61.0 \pm 18.8$ & $77.6 \pm 16.0$ & $<0.0001^{\star \star \star}$ \\
\hline Sleep/energy domain & $64.9 \pm 18.6$ & $77.4 \pm 17.7$ & $0.0003^{* * *}$ \\
\hline Bother/concern domain & $60.6 \pm 21.5$ & $79.1 \pm 15.1$ & $<0.0001^{\star \star \star}$ \\
\hline Global health status & $5.0 \pm 2.9$ & $2.9 \pm 2.4$ & $<0.0001^{* * *}$ \\
\hline PSQ। & $9.2 \pm 2.5$ & $7.2 \pm 3.2$ & $0.0022^{\star \star}$ \\
\hline \multicolumn{4}{|c|}{ Differences compared using the Wilcoxon signed rank test } \\
\hline$† 50 \mathrm{mg}$ of oral mirabegron once daily & & & \\
\hline
\end{tabular}

compliance and adverse events were recorded.

The primary endpoint was a change in the nocturnal voiding frequency as recorded in the voiding diary. The secondary endpoints were changes in the following: the total score and subscale scores on the N-QOL and global health status; total score and individual symptom scores on the OABSS; total score and scores for voiding, storage, and post-micturition symptoms on the I-PSS; the I-PSSQOL; and the PSQI.

Based on the results recorded in the voiding diary, changes over time in the following parameters were investigated: voiding frequency per day, 24-h voiding volume, urine volume per void, daytime voiding frequency, day time urine volume, nocturnal voiding frequency, nocturnal urine volume, nocturnal polyuria index, urgency episodes per day, urgency incontinence episodes per day, Hours of Undisturbed Sleep (HUS), and maximum bladder capacity.

For statistical analyses, the values of each parameter before and after mirabegron administration were compared using the Wilcoxon signed rank test or the paired t-test. The significance level was set at 0.05 .

\section{Results}

In total, 58 patients were analyzed (mean age, $75.3 \pm 6.8$ years) after 2 patients were excluded because they withdrew consent.

Changes in nocturia as recorded in the voiding diary for each patient and changes in individual parameters on the voiding record are shown in (Table 1). The nocturnal voiding frequency significantly decreased from $2.5 \pm 1.1$ times per night before treatment to $1.9 \pm 1.0$ times per night after 12 weeks of treatment. In addition, significant improvements were observed in the daytime voiding frequency and the 24 -h voiding frequency after 12 weeks of treatment. There were no significant changes in the urine volume parameters before and after treatment (24-h urine volume, daytime urine volume, and nocturnal urine volume). There was also no significant change in the nocturnal polyuria index after treatment. However, there was a significant increase in both the day time and night time urine volume per void after 12 weeks of treatment. In addition, the urine volume of the first nocturnal void significantly increased from $184.5 \pm 83.6 \mathrm{~mL}$ before treatment to $228.1 \pm 110.2 \mathrm{~mL}$ after 12 weeks of treatment. The mean HUS was extended by $\geq 40$ min after 12 weeks of treatment, although this difference in HUS before and after treatment was not significant (Table 1).
The questionnaire results and changes in the I-PSS and OABSS before and after treatment are shown in (Table 2). The total I-PSS and the I-PSS for voiding, storage, and post-micturition symptoms were significantly improved after 12 weeks of treatment. The I-PSS nocturia symptoms significantly improved from $2.5 \pm 0.9$ points before treatment to $1.9 \pm 0.8$ points after 12 weeks of treatment. The I-PSS-QOL score also significantly improved from $4.8 \pm 1.1$ points before treatment to $3.0 \pm 1.5$ points after 12 weeks of treatment. The total OABSS and the subscales of individual OAB symptoms were also significantly improved after 12 weeks of treatment; the nocturia score significantly improved from $2.5 \pm 0.8$ points before treatment to $1.8 \pm 0.8$ points after 12 weeks of treatment.

Table 3 shows the N-QOL scores and the PSQI before and after treatment. The total N-QOL score significantly improved from 61.0 \pm 18.8 points before treatment to $77.6 \pm 16$.0points after 12 weeks of treatment, with significant improvements in the two subscale domains of sleep/energy and bother/concern. The global health status also significantly improved from $5.0 \pm 2.9$ points before treatment to $2.9 \pm 2.4$ points after 12 weeks of treatment. For the PSQI, 30 patients with a score of $\geq 5.5$ points (suggestive of a sleep disorder) before treatment were evaluated; the PQSI significantly improved from 9.2 \pm 2.5 points before treatment to $7.2 \pm 3.2$ points after 12 weeks of treatment.

Adverse events possibly related to mirabegron occurred in five patients (8.3\%). These adverse events were dry mouth in two patients, constipation in one patient, diarrhea in one patient, and palpitations in one patient; all adverse events were mild in severity and did not caused is continuation of treatment.

\section{Discussion}

In the present study, we evaluated the efficacy and safety of mirabegron for nocturia in female patients with $\mathrm{OAB}$. Many previous studies have indicated the efficacy of mirabegron for $\mathrm{OAB}$ symptoms [14-18], but these studies used the voiding frequency and urinary incontinence frequency as the primary endpoints. Few studies have used the nocturnal voiding frequency as a primary endpoint in patients with nocturia. Based on the voiding diary entries in the present study, 12 weeks of mirabegron administration significantly reduced the nocturnal voiding frequency, which was the primary endpoint in this study. This decrease in nocturnal voiding frequency was also seen in the nocturia parameters of the I-PSS and the OABSS (secondary endpoints); hence, the effect of mirabegron on nocturia 
was confirmed by multiple parameters. In addition, 12 weeks of mirabegron administration significantly improved OAB symptoms other than the nocturnal voiding frequency, which is similar to the findings of previous studies [14-18].

Nocturia considerably compromises QOL $[3,4]$. The diseasespecific QOL questionnaire for nocturia is the N-QOL. The usefulness of this questionnaire has been recognized by the International Consultation on Incontinence [19,20], and a Japanese version of the N-QOL has also been developed [21,22]. In a recent study, oral administration of $25 \mu \mathrm{g}$ desmopressin significantly reduced the nocturnal voiding frequency in female patients with two or more nocturnal voids per night, with a significant improvement in the $\mathrm{N}-\mathrm{QOL}$ score [23]. In addition, there was a significant decrease in the nocturnal voiding frequency and significant improvements in the $\mathrm{N}$-QOL scores after administration of imidafenacin in male patients with lower urinary tract symptoms in whom $\mathrm{OAB}$ had persisted after administration of anal-blocker for $\geq 1$ month [24]. However, no study has evaluated the effects of mirabegron on nocturia using the N-QOL as an indicator. The present study demonstrated that mirabegron significantly reduced the nocturnal voiding frequency in female patients with nocturia, and the significant improvement in the $\mathrm{N}-\mathrm{QOL}$ contributed to the overall improvement in QOL in patients with nocturia.

The present study also revealed that mirabegron significantly improved the PSQI, a sleep disorder index; this suggests that mirabegron is also effective for sleep disorders. The HUS is a useful indicator in evaluating the quality of sleep $[25,26]$. The HUS reportedly improves with the improvement of nocturia by administration of anticholinergic drugs $[27,28]$; the results of the present study suggest that mirabegron, similarly to anticholinergic drugs, could prolong the HUS by improving the nocturnal voiding frequency, thus contributing to improved quality of sleep and reduction of sleep disorder symptoms.

The main mode of action of mirabegron is that it binds to $\beta 3$ receptors in the smooth muscle of the bladder to relax the muscle, leading to an increase in bladder capacity [13]. It also significantly increased the voided volume during the day and night in the present study; this effect may be based on the ability of mirabegron to relax the bladder smooth muscle, resulting in a reduction of the voiding frequency. In addition, the urine volume of the first nocturnal void significantly increased, suggesting that this may be related to the prolonged HUS. A recent report revealed that some anticholinergic drugs effectively reduce the nocturnal urine volume and the nocturnal polyuria index and that this effect may be involved in the reduced nocturnal voiding frequency [28]. However, the detailed mechanism of action has not been elucidated. In the present study, mirabegron neither significantly changed the voided volume during the day or night nor changed the nocturnal polyuria index, which suggests that mirabegron had no effect on reducing the nocturnal urine volume.

The major limitation of the present study was that it was not a placebo-controlled trial with comparison between treatment and control groups. In addition, the study included a limited number of patients. However, this study was grounded in actual clinical practice, and the results support the usefulness of mirabegron for nocturia. A large-scale, placebo-controlled, randomized trial is required in the future. Furthermore, this study was conducted in only female patients, and the effects of mirabegron on nocturia in male patients were not evaluated. Male patients were excluded because older men often develop lower urinary tract obstruction such as that caused by prostatic hyperplasia, and they therefore have a more complicated pathology than females. Future investigation of male patients with nocturia is required.

Many factors are involved in the development of nocturia, and nocturnal polyuria is one of the major factors. In this study, polyuric patients with mean daily voided volume of $\geq 40 \mathrm{~mL} / \mathrm{kg}$ were excluded, but patients with nocturnal polyuria were not excluded. Thus, the nocturnal polyuria index before treatment was $40.3 \%$, and more than half of the participants had nocturnal polyuria. Although it is presumed from the results of this study that mirabegron may also be effective in some patients with nocturnal polyuria, further investigation of the effects of mirabegron in these patients is required.

\section{Conclusion}

The present study showed that mirabegron, a $\beta 3$-adrenoceptor agonist, improved the nocturnal voiding frequency by increasing the nocturnal bladder capacity and thereby improved the QOL by increasing sleep quality in female patients with $\mathrm{OAB}$ and nocturia.

\section{Acknowledgment}

The authors greatly appreciate the following doctors of the Study Group of N-QOL for their evaluation of the study protocol and accumulation of cases: Dr. MotohiroSenda, Dr. Toshikazu Otani, Dr. Yuichi Sai, Dr. Shiko Kai, Dr. Masayuki Otani, Dr. Kuniaki Tanaka, Dr. Yoshikazu Tsuji, Dr. Harunori Narita, Dr. Hideki Mizuno, Dr. Shutoku Sai, and Dr. Shin'ichiTakamura.

\section{Disclosure}

This study was funded by Astellas Pharma Inc. Astellas Pharma Inc. had no role in the study design or the collection, analysis, or interpretation of data.

MY is a consultant for Astellas Pharm Inc. and has received lecture fees from Astellas Pharm Inc.MG is a consultant for Astellas Pharm Inc. and has received lecture fees and research contribution from Astellas Pharm Inc. SK has received lecture fees from Astellas Pharm Inc. KK has received lecture fees from Astellas Pharm Inc. MN is a consultant for Astellas Pharm Inc. and has received lecture fees from Astellas Pharm Inc. YM has no conflict of interest.

\section{References}

1. Abrams P, Cardozo L, Fall M, Griffiths D, Rosier P, Ulmsten U, et al. The standardisation of terminology of lower urinary tract function: report from the Standardisation Sub-Committee of the International Continence Society. Neurourol Urodyn. 2002; 21: 167-178.

2. Weiss JP, Blaivas JG, Stember DS, Brooks MM. Nocturia in adults: etiology and classification. Neurourol Urodyn. 1998; 17: 467-472.

3. Coyne KS, Zhou Z, Bhattacharyya SK, Thompson CL, Dhawan R, Versi E. The prevalence of nocturia and its effect on health-related quality of life and sleep in a community sample in the USA. BJU Int. 2003; 92: 948-954.

4. Asplund R, Marnetoft SU, Selander J, Akerström B. Nocturia in relation to somatic health, mental health and pain in adult men and women. BJU Int. 2005; 95: 816-819.

5. Homma Y, Yamaguchi O, Hayashi K. Neurogenic Bladder Society Committee. 
Epidemiologic survey of lower urinary tract symptoms in Japan. Urology. 2006; 68: 560-564.

6. Brubaker L, Fitzgerald MP. Nocturnal polyuria and nocturia relief in patients treated with solifenacin for overactive bladder symptoms. Int Urogynecol Pelvic Floor Dysfunct. 2007; 18: 737-741.

7. Rudy D, Cline K, Harris R, Goldberg K, Dmochowski R. Multicenter phase III trial studying trospium chloride in patients with overactive bladder. Urology. 2006; 67: 275-280.

8. Nitti VW, Dmochowski R, Appell RA, Wang JT, Bavendam T, Guan Z Efficacy and tolerability of tolterodine extended-release in continent patients with overactive bladder and nocturia. BJU Int. 2006; 97: 1262-1266.

9. Nomiya M, Yamaguchi O. A quantitative analysis of mRNA expression of $\alpha 1$ and $\beta$-adrenoceptor subtypes and their functional roles in human normal and obstructed bladders. J Urol. 2003; 170: 649-653.

10. Igawa $\mathrm{Y}$, Yamazaki $\mathrm{Y}$, Takeda $\mathrm{H}$, Hayakawa K, Akahane M, Ajisawa Y, et al Functional and molecular biological evidence for a possible $\beta 3$-adrenoceptor in the human detrusor muscle. Br J Pharmacol. 1999; 126: 819-825.

11. Takeda M, Obara K, Mizusawa T, Tomita Y, Arai K, Tsutsui T, et al. Evidence for $\beta 3$-adrenoceptor subtypes in relaxation of the human urinary bladder detrusor: analysis by molecular biological and pharmacological methods. J Pharmacol Exp Ther. 1999; 288: 1367-1373.

12. Fujimura T, Tamura K, Tsutsumi T, Yamamoto T, Nakamura K, Koibuchi $Y$, et al. Expression and possible functional role of the $\beta 3$-adrenoceptor in human and rat detrusor muscle. J Urol. 1999; 161: 680-685.

13. Yamaguchi O. Beta 3-adrenoceptors in human detrusor muscle. Urology 2002; 59: 25-29.

14. Yamaguchi O, Marui E, Kakizaki H, Homma Y, Igawa $\mathrm{Y}$, Takeda M, et al Phase III, randomised, double-blind, placebo-controlled study of the $\beta 3$ adrenoceptor agonist mirabegron, $50 \mathrm{mg}$ once daily, in Japanese patients with overactive bladder. BJU Int. 2014; 113: 951-960

15. Khullar V, Amarenco G, Angulo JC, Cambronero J, Høye K, Milsom I, et al. Efficacy and tolerability of mirabegron, a $\beta 3$-adrenoceptor agonist, in patients with overactive bladder: results from a randomised European-Australian phase 3 trial. Eur Urol. 2013; 63: 283-295.

16. Nitti VW, Auerbach S, Martin N, Calhoun A, Lee M, Herschorn S. Results of a randomized phase III trial of mirabegron in patients with overactive bladder. J Urol. 2013; 189: 1388-1395.

17. Herschorn S, Barkin J, Castro-Diaz D, Frankel JM, Espuna-Pons M, Gousse $A E$, et al. A phase III, randomized, double-blind, parallel-group, placebocontrolled, multicentre study to assess the efficacy and safety of the $\beta 3$ adrenoceptor agonist, mirabegron, in patients with symptoms of overactive bladder. Urology. 2013; 82: 313-320.
18. Nitti VW, Khullar V, Van Kerrebroeck P, Herschorn S, Cambronero J, Angulo $\mathrm{JC}$, et al. Mirabegron for the treatment of overactive bladder: a pre specified pooled efficacy analysis and pooled safety analysis of three randomised, double-blind, placebo-controlled, phase III studies. Int J Clin Pract. 2013; 67 : 619-632.

19. Abraham L, Hareendran A, Mills IW, Martin ML, Abrams P, Drake MJ, et al. Development and validation of quality-of-life measure for men with nocturia. Urology. 2004; 63: 481-486

20. Abrams P, Avery K, Gardener N, Donovan J. ICIQ Advisory Board. The International Consultation on Incontinence Modular Questionnaire. J Urol. 2006; 175: 1063-1066.

21. Yoshida M, Gotoh M, Homma Y, Honda K, Nomiya M, Yamaguchi O, et al. Development and linguistic validation of the Japanese version of the Nocturia Quality of Life Questionnaire (N-QOL). Journal of the Japan Neurogenic Bladder Society. 2009; 20: 317-324.

22. Ikeda S, Kobayashi M, Shimizu T, Yoshida M, Gotoh M, Homma Y, et al. Evaluation of psychometric properties of the Japanese version of the Nocturia Quality of Life Questionnaire (N-QOL). Journal of the Japan Neurogenic Bladder Society. 2009; 20: 325-331.

23. Sand PK, Dmochowski RR, Reddy J, van der Meulen EA. Efficacy and safety of low dose desmopressin orally disintegrating tablet in women with nocturia: results of a multicenter, randomized, double-blind, placebo controlled, parallel group study. J Urol. 2013; 190: 958-964.

24. Yokoyama O, Tsujimura A, Akino H, Segawa N, Tamada S, Oguchi N, et al. Add-on anticholinergic therapy for residual nocturia in patients with lower urinary tract symptoms receiving a1-blocker treatment: a multi-centre, prospective, randomised study. World J Urol. 2015; 33: 659-667.

25. Chartier-Kastler E, Chapple CR. LUTS/BPH in clinical practice: the importance of nocturia and quality of sleep. BJU Int. 2006; 98: 3-8.

26. Chartier-Kastler E, Tubaro A. The measurement of nocturia and its impact on quality of sleep and quality of life in LUTS/BPH. Eur Urol Suppl. 2006; 5: 3-11.

27. Yokoyama O, Yamaguchi O, Kakizaki H, Itoh $\mathrm{N}$, Yokota $\mathrm{T}$, Okada $\mathrm{H}$, et al. Efficacy of solifenacin on nocturia in Japanese patients with overactive bladder: impact on sleep evaluated by bladder diary. J Urol. 2011; 186: 170174.

28. Yokoyama O, Homma Y, Yamaguchi O. Imidafenacin, an antimuscarinic agent, improves nocturia and reduces nocturnal urine volume. Urology. 2013; 82: $515-520$
Austin J Urol - Volume 4 Issue 1 - 2017

ISSN: 2472-3606 | www.austinpublishinggroup.com

Yoshida et al. (C) All rights are reserved
Citation: Yoshida M, Gotoh M, Kageyama S, Kato K, Matsukawa Y, Narushima M. Mirabegron Improves Nocturia, Nocturia-Associated Quality of Life, and Sleep Quality in Female Patients with Overactive Bladder. Austin J Urol. 2017; 4(1): 1054 